\title{
Proyecto Construcción de Zonas de Paz: análisis de la percepción de la niñez y la adolescencia sobre la violencia y la paz en la comunidad de Guararí
}

\section{Construction of Peace Zones Project: Analysis of the perception of girls and adolescents about violence and peace in the community of Guararí.}

\section{Projeto Construção de Zonas de Paz: Análise da percepção de crianças e adolescentes sobre violência e paz na comunidade de Guararí.}

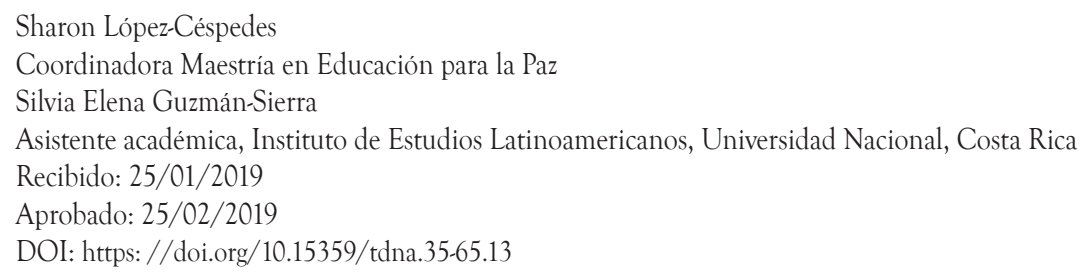

Sharon López-Céspedes

Coordinadora Maestría en Educación para la Paz

Silvia Elena Guzmán-Sierra

Asistente académica, Instituto de Estudios Latinoamericanos, Universidad Nacional, Costa Rica

Recibido: 25/01/2019

Aprobado: 25/02/2019

DOI: https: //doi.org/10.15359/tdna.35-65.13

\section{Resumen}

El proyecto Construcción de Zonas de Paz: análisis de la percepción de la niñez y la adolescencia sobre la violencia y la paz en la comunidad de Guarari se enmarca en los esfuerzos que la Universidad $\mathrm{Na}$ cional, a través de la Vicerrectoría de Extensión, viene realizando en el Proyecto Ciudades Culturales, las cuales, en conjunto con la Municipalidad de Heredia y el Centro Cívico por la $\mathrm{Paz}$ (CCP) de Guararí, articulan procesos para la prevención de la violencia y la construc-

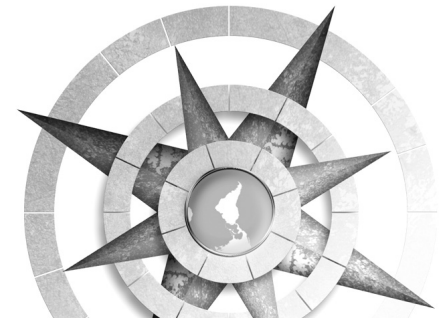

ción de la paz enfocados, principalmente, en la niñez, la adolescencia y la juventud en riesgo.

Palabras clave: zonas de paz, violencia y paz, prevención de la violencia, Guararí, Instituto de Estudios Latinoamericanos

\section{Abstract}

Building Zones of Peace (BZP) Project: Analysis of the perception of girls and adolescents about violence and peace in the community of Guarari is part of the efforts made by the Universidad Nacional, through the Office of Extension, in carrying out the "Cultural Cities" project, which together with the Municipality of Heredia and the Civic Center for Peace (CCP) of Guararí, articulate processes for the prevention of violence and the construction of peace, focused mainly on children, adolescents and youth at risk. 
Keywords: Zones of Peace, violence and peace, prevention of violence, Guararí, Instituto de Estudios Latinoamericanos (IDELA)

\section{Resumo}

O Projeto de Construção de Zonas de Paz: A análise da percepção de crianças e adolescentes sobre violência e paz na comunidade de Guarari faz parte dos esforços que a Universidade Nacional, através do $\mathrm{Vi}$ certório de Extensão, está realizando no Projeto Cidades Culturais, que, juntamente com a Prefeitura de Heredia e o Centro Cívico da Paz (CCP) de Guararí, articulam processos para a prevenção da violência e a construção da paz voltados principalmente para crianças, adolescentes e jovens em situação de risco.

Palavras chave: Zonas de paz, violência e paz, prevenção da violência, Guarari, Instituto de Estudos Latino-Americanos

Conjuntamente con la Vicerrectoría de Extensión, la Municipalidad de Heredia y el Centro Cívico para la Paz de Guararí, el Instituto de Estudios Latinoamericanos (IDELA), referente regional en la formación en el nivel de posgrado en derechos humanos y educación para la paz, privilegia espacios donde pueda liderar proyectos que, además de potenciar la construcción del conocimiento y el aprendizaje vivencial de sus estudiantes, generen un impacto positivo en las comunidades, particularmente, en aquellos grupos poblacionales que se encuentran en situaciones de mayor vulnerabilidad, como es la población primaria de esta propuesta de atención integral del conflicto, la violencia y la construcción de la paz social.

La comunidad de Guararí está ubicada en el distrito de San Francisco del cantón central de la provincia de Heredia y se considera la zona de mayor vulnerabilidad social de dicho cantón .Esto se da como producto del desarrollo de proyectos de vivienda de interés social, que a su vez incide en una serie de factores como la segregación social por la concentración de familias de bajos ingresos socioeconómicos, la deficitaria presencia estatal, el aumento de la población migrante, entre otros ${ }^{1}$.

Resultado de lo anterior, se ha generado una serie de patologías sociales: problemas asociados al trasiego y consumo de drogas, delincuencia, criminalidad, hacinamiento, embarazos en adolescentes, falta de tenencia de vivienda digna, violencia intrafamiliar y deserción escolar. ${ }^{2}$ Esta situación que ha llevado a considerar a Guararí entre las áreas con mayores índices de violencia del país, misma razón por la cual fue seleccionada para la construcción de un CCP, que plantea

1 Ver Chávez, Diagnóstico. 2014. Heredia: Centro Cívico por la Paz Guararí. Documento interno de trabajo.

2 Ver Chávez, Diagnóstico.2014. Heredia: Centro Cívico por la Paz Guararí. Documento interno de trabajo.
184 Proyecto Construcción de Zonas de Paz: análisis de la percepción de la niñez y la adolescencia sobre la violencia y la paz en la comunidad de Guararí

López-Céspeded/Guzmán-Sierra 
una estrategia de intervención en lo local, con el fin de prevenir la violencia. De esta forma, los CCP establecen como prioridad atención a la población de niños y los adolescentes específicamente aquella, entre 13 y 19 años.

En consecuencia, el Proyecto Construyendo Zonas de Paz tiene como objetivo fortalecer las capacidades para la transformación de los conflictos, de este modo contribuir a la reducción de la violencia y la construcción de la paz. Para ello contempla una estrategia pedagógica integral desde un enfoque de educación para la paz y los derechos humanos, dirigida a trabajar con población adolescente-joven.

En un sentido teórico y práctico, se entiende que tanto la paz como la violencia no son estados permanentes o estáticos, sino que ambos son procesos sociales que se van entrecruzando de manera compleja y se ven atravesados por múltiples factores y contextos.

Distinguimos en este procedimiento complejo tres formas de violencia: la violencia directa, la cual tendría implicaciones materiales directas sobre los cuerpos, la naturaleza o los bienes; la estructural que devendría en la institucionalizada (instituciones, sistemas políticos, organización social), y la cultural, el gran marco en el que las anteriores son legitimadas, justificadas y normalizadas.
La paz se entiende como un proceso construido en lo cotidiano, desde la creatividad de las personas, y que puede ser edificada en el nivel de lo directo, lo estructural o lo cultural. Por tanto, la paz no sería la ausencia absoluta de conflicto o un estado permanente, sino, más bien, las opciones de vida cimentadas individual o colectivamente y que hacen frente a las violencias, con el objetivo de crear alternativas autónomas de dignidad. Así, el trabajo por la paz lo podemos traducir como espacios que brindan la posibilidad de construir diálogos culturales, sociales y comunitarios, esos donde todas las personas tienen voz.

La construcción de formas pacíficas de convivencia, en espacios donde la violencia ha sido instalada y normalizada, es compleja y requiere el análisis de sus múltiples factores. El proceso de formación de la paz tiene como reto de la elaboración creativa, ante un sistema de patrones violentos autoperpetuables, reproducidos por una multifactorialidad intrincada en las relaciones sociales. En ese sentido, nuestra intención se orienta a que los participantes del proyecto se entiendan precisamente, como agentes transformadoras de las condiciones de violencia tanto imaginativas como creativas.

Con estas consideraciones se construyó un proceso participativo en el cual los integrantes de Construyendo Zonas de $\mathrm{Paz}$, así como niñas del proyecto Liga

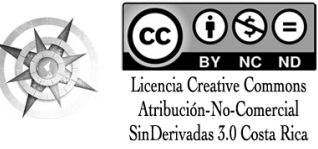


Fem: la cancha para nosotras, de Sepro Joven $^{3}$ (en suma 18 niñas y adolescentes entre los 7 y 16 años), acompañados por 6 estudiantes de la Maestría en Derechos Humanos y Educación para la Paz del IDELA, emprendieron un camino para plasmar, a través de fotografías, lo que ellos identifican como zonas de paz y de violencia en su comunidad.

En un primer momento, el equipo de estudiantes de la maestría facilitó 2 encuentros para la revisión participativa de los conceptos de violencia directa, estructural y cultural; esto con el fin de que, una vez identificados tales conceptos, los participantes pudieran determinar zonas de paz y de violencia en su comunidad. En un segundo momento, los integrantes realizaron una cartografía simbólica de su comunidad, estableciendo lo que para ellos generaba paz o producía violencia. En un tercer momento, y habiendo identificado los diferentes lugares, las personas participantes llevaron a cabo un trabajo de campo en el que tomaron fotografías de los lugares identificados en las cartografías.

Esta experiencia tuvo como producto artístico la exposición fotográfica Paz y

3 Esta alianza se da puesto que el proyecto Construyendo Zonas de Paz se desarrolló durante el 2017, en el Centro Cívico por la Paz de la comunidad de Guararí de Heredia, Costa Rica, lugar donde también toma espacio el proyecto Liga Fem: la cancha para nosotras de Sepro Joven.
Violencia en Nuestra Comunidad 4 . La selección de 12 fotografías se hizo de entre más de 200 tomadas por las 11 personas participantes, las cuales en un proceso participativo, reflexivo y dialógico, en el que expresaban sus percepciones sobre las violencias y la paz, así como escogían aquellas fotografías más representativas de sus ideas y sentires.

De este trabajo, se recogen 3 resultados referentes a formas de violencia mayormente identificadas por las personas participantes; estas son: la violencia contra la naturaleza (contaminación) y el uso de los espacios públicos, la socioeconómica (desigualdad y pobreza) y aquella contra las mujeres. Los participantes reconocen también formas de paz, en las cuales se manifiesta claramente cómo los vínculos con la naturaleza, así como la inclusión social y los espacios recreativos les generan la posibilidad de la construcción de su propia paz. Un análisis de estas 3 formas de violencia y su impacto sobre dicha población, así como de la construcción de las formas de paz será abordado a continuación.

4 Boletín informativo del proyecto redactado por los participantes. https://es.calameo.com/ $\mathrm{read} / 0052319187323027368 \mathrm{cb}$ Afiche de la exposición fotográfica: https://www.facebook.com/infolatino. idela/photos/a.972486082832286.1073741828. 972427676171460/1578810378866517/ ?type $=3 \&$ theater 
Las personas participantes, a través de su investigación de campo, identifican diferentes zonas en la comunidad marcadas por la contaminación; entre las cuales se encuentran lotes baldíos, quebradas, aceras y alcantarillas.

La visita a estos sitios permite tres reflexiones importantes. Como primera reflexión los participantes analizan el problema de la basura, cómo la acumulación de esta en las aceras conlleva que las alcantarillas se taqueen y, en época lluviosa se produzcan inundaciones que, como consecuencia, traen perjuicios económicos a las familias. De la misma manera, se hace evidente que la contaminación por sólidos en su comunidad afecta los ríos cercanos y elimina posibles fuentes de agua potable. Este análisis facilitó reflexionar sobre la interdependencia y complementariedad de los derechos humanos. Resulta indudable para ellos que la violencia contra la naturaleza limita su derecho a un ambiente sano, pero también a una vivienda digna así como al consumo de agua potable y que dicha contaminación genera repercusiones económicas para las familias.

Una segunda reflexión refiere a la responsabilidad sobre el manejo de los desechos. Por un lado, se evidencia el compromiso del gobierno local en su gestión, en cuanto a la recolección de basura. En este sentido, señalan 2 factores deficientes: el primero de ellos es relativo a la cantidad de veces a la semana que se realiza el servicio de recolección y el segundo, a la calidad de este. Las personas participantes denuncian que la recolección se hace de manera apresurada y que, en ocasiones, algunos residuos no se recogen, por lo que se acumulan en las aceras y las calles; sin embargo, también apuntan la responsabilidad ciudadana en el manejo de los residuos, pues, según sus apreciaciones algunos habitantes de la comunidad no separan sus residuos, no desechan la basura en los basureros públicos y no cierran bien las bolsas o las dejan al alcance de los animales y estos esparcen la basura. Los participantes concluyen que, si bien los servicios municipales no son los más adecuados, mucha de la contaminación podría ser evitada si los ciudadanos actuaran de manera más sensata.

Una tercera reflexión, más que un análisis de la realidad o una denuncia por la responsabilidad en torno al tema de la violencia contra la naturaleza, es un reclamo cargado de esperanza. Las personas participantes denotan los espacios públicos abandonados y ociosos; frente a ello, denuncian la falta de estos para la recreación de la juventud. Una de las participantes inclusive ahonda más en la crítica y hace referencia a cómo los adolescentes no tienen espacios públicos para la recreación, el disfrute y la socialización. Ella evidencia como los pocos lugares que existen para este fin están destinados la niñez y población 
adolescente es expulsada de manera normativa y, principalmente, de modo social de estos espacios. Este reclamo comprueba de nuevo cómo la cooptación de un derecho, el del ambiente sano perjudica el acceso a otro, el del al ocio y de la recreación. Estamos ante lo que Steven Flusty describe como "espacios prohibitorios [...], diseñados para interceptar y rechazar o filtrar a los que aspiran usarlos" (Bauman, 2001: p. 31).

El uso del espacio público queda doblemente inaccesible para las personas adolescentes, en primer lugar, por su, desuso y abandono y, en segundo lugar, debido a que aquellos posibles sitios de recreación se entienden como excluyentes para personas con sus cualidades etarias. Las posibilidades de identificación socio espacial que puedan tener las personas con los espacios comunitarios son ínfimamente reducidas, pues no hay una experimentación del ámbito público como propio, limitándose las relaciones sociales a lo privado y familiar, y ambientes que las personas identifican como de paz (especialmente sus habitaciones). Sin embargo, esta desvinculación con lo comunitario podría decantar en la desconexión con las problemáticas y necesidades comunitarias.

La reducción de los espacios públicos es también una de los políticos y comunales ${ }^{5}$; por lo tanto, estos espacios que

5 El filósofo Zigmund Bauman señala que, en la contemporaneidad globalizante, los espacios públicos, podrían facilitar una comprensión de lo que es una ciudadanía activa quedan fuera del alcance de las personas jóvenes. Los ambientes públicos resultan residuales e impotentes. No obstante, ante esta situación, las personas participantes se llenan de esperanza y ven en la organización comunitaria, la toma y el embellecimiento de los espacios públicos una posibilidad para el disfrute de la niñez y la adolescencia. Ellos se entienden como sujetos de derechos y por tanto como tales reclaman la universalidad de estos, entendiéndola en función del

fortalecimiento de individuos, grupos y organizaciones a la hora de construir un marco de acción que permita a todos y a todas ir creando las condiciones que garanticen de un modo igualitario su acceso a los bienes materiales e inmateriales que hacen que la vida sea digna de ser vivida (Herrera Flores, 2001: p. 13).

El análisis de la violencia contra la naturaleza hecha por los constructores de paz de la comunidad de Guararí recuerda la premisa de que los derechos humanos son universales y, por tanto indivisibles

ágoras y foros en sus diversas expresiones [...] siguieron a la élite al liberarse de sus anclajes locales; son los primeros en desterritorializarse y ponerse fuera del alcance de la capacidad comunicativa del factor humano de una localidad y sus residentes. Lejos de engendrar comunidades las poblaciones locales son como haces de cabos sueltos (Bauman, 2001, pág. 35).
188 Proyecto Construcción de Zonas de Paz: análisis de la percepción de la niñez y la adolescencia sobre la violencia y la paz en la comunidad de Guararí López-Céspedes/ Guzmán-Sierra 
los unos de los otros, pero además, que son una constante lucha por la dignidad de las personas, las cuales toman las indispensables formas materiales en las oportunidades y condiciones para el acceso a bienes y servicios.

Las propuestas de embellecimiento de la comunidad hechas por las personas participantes son un ejemplo de lucha llena de esperanza y creatividad. Necesitamos, pues, recomponer espacios o zonas de informalidad, los cuales propongan visiones alternativas y antisistémicas que tengan como objetivo ir construyendo las bases sociales y culturales de una nueva hegemonía. Por ello, para conocer y practicar los derechos, es preciso situarlos en esos espacios simbólicos en los que las relaciones de poder colocan a los individuos en diferentes planos desde los que es posible alcanzar la dignidad 6 .

Quizá la violencia socioeconómica para una comunidad como Guararí de Heredia sea una de las formas más evidentes y cotidianas en las vidas de quienes la habitan. Sin embargo un fenómeno como la pobreza estructural es tan complejo que una investigación sencilla como la que hicieron los constructores de paz pondrá en evidencia sus consecuencias más subjetivas.

Un primer elemento que llama la atención es cómo estratifican los barrios

6 Ver Herrera Flores, La reinvención de los derechos humanos, p. 128. de la comunidad, dependiendo de la condición socioeconómica de estos. Los participantes hacen un ejercicio espontáneo de asignación de los estigmas culturales impuestos a los habitantes de Guararí por las personas de la comunidad herediana y nacional, quienes pueblan ciertos barrios específicos de la zona y no a la totalidad de ella. Un ejemplo es cómo algunos de quienes viven en Guararí consideran que es el área de La Milpa la que es violenta, cayendo en la misma generalización de la que ellos mismos son víctimas.

Un análisis de estos estigmas y de la visibilización de las diferencias socioeconómicas entre ellos mismos permite la generación de conciencia sobre la desigualdad y un examen de sus causas y consecuencias, que se desarrolla, principalmente, en torno al derecho a una vivienda digna.

Otro elemento que sobresale es el de los vínculos socioafectivos creados por algunas de las participantes hacia instituciones concretas que en el pasado les han provisto de ayudas económicas para la satisfacción de sus necesidades. Estos lugares toman un espacio fundamental en sus historias de vida, pues se entiende que, de no ser por ciertas colaboraciones su derecho a la educación o a la alimentación no hubiese podido ser subsanado. Es importante destacar una diferenciación etaria con respeto a este vínculo socioafectivo entre las personas participantes y las instituciones, 
porque pues pareciera que, a menor edad ellos confían más en las instituciones de asistencia como el Instituto Mixto de Ayuda Social (IMAS). Aun así creen de manera más esperanzada en la autogestión comunitaria, mientras que, a mayor edad, las personas dejan de tener confianza en las instituciones $y$, sin embargo, les relegan a estas, sin optimismo, las responsabilidades sociales y comunitarias.

El análisis de la violencia socioeconómica es sumamente complejo y está cargado de la sensibilidad que deviene de una vida de imposiciones culturales negativas hacia la población de Guararí. No obstante, en resistencia ante esa violencia, los participantes son conscientes de que su comunidad, a pesar de estar marcada por la agresión y la desigualdad, es un lugar "con gente buena" y diversa. Ellos, a través de un ejercicio bondadoso, valoran las diferencias haciendo consciencia de la inmaterialidad, abstracción y absolutización tanto de los estigmas como de los prejuicios sociales y, ante ello, validan la dignidad propia de la diversidad, se reconocen en la periferia y desde ese lugar enuncian.

Por esta razón, la visión compleja de los derechos apuesta por situarnos en la periferia. Centro solo hay uno y aquello que no coincida con este es abandonado a la marginalidad. Por el contrario, las periferias son muchas. En realidad todo es periferia, si aceptamos que no hay nada puro y que todo está relacionado?

La tarea es, pues, desde los lugares de la alteridad, reflexionar, buscar soluciones locales materiales e inmateriales para la construcción de identidades y vidas dignas. A este respecto, uno de los principales razonamientos hechos por los participantes es que, a pesar de las discriminaciones que la mayoría de ellos, y sus familias experimentan por su origen migrante o condición socioeconómica, estas supuestas diferencias ante los otros (personas externas a la comunidad) pueden ser subsanadas con diálogos interculturales y empatía.

Los participantes emprenden una denuncia desde el reconocimiento mutuo, desde lo impuro y la mezcla. Entienden la diversidad que conlleva la posibilidad intersubjetiva de dignificación, y así comprenden que la violencia socioeconómica (la jerarquía) es la estructura de poder que impone las diferencias (narraciones de la estratificación barrial), pero que, en el reconocimiento de la dignificación y las distinciones que subsisten, existe la posibilidad de vinculación para construir la paz en su comunidad y eliminar los estigmas sociales asociados a las personas habitantes de Guararí.

7 Ver Herrera Flores, La reinvención de los derechos humanos, p. 145.
190 Proyecto Construcción de Zonas de Paz: análisis de la percepción de la niñez y la adolescencia sobre la violencia y la paz en la comunidad de Guararí

López-Céspedes/ Guzmán-Sierra 
A diferencia de las conclusiones más prácticas con respecto a la capacidad de acción directa frente a la violencia contra la naturaleza y el uso de espacios públicos, las personas participantes disciernen que sus capacidades de acción frente a la violencia socioeconómica son más limitadas, sin embargo es fundamental la toma de consciencia y el posicionamiento que ellos toman, entendiendo que, frente a la violencia estructural, denunciar las desigualdades y encontrar vías locales para que la estructura violenta no se imponga en la construcción de identidad de los sujetos es un ejercicio revolucionario.

Por otro lado, las diversas formas en las que la violencia contra las mujeres atraviesa las vidas de los participantes ha sido un elemento traído a la discusión de manera recurrente por parte de las personas involucradas, a lo largo del proceso. Estas en comparten situaciones de violencia en sus casas, en sus comunidades y en el colegio, vividas por ellas mismas, sus pares o familiares, entre las que están el abuso sexual; acoso callejero o escolar; abuso físico, psicológico y patrimonial.

Propiamente, durante el proceso de elaboración de la cartografía simbólica de las violencias, se plasman diferencias en la forma en que los participantes, tanto las niñas, como los adolescentes, entienden y reflexionan sobre la agresión contra las mujeres, según sus edades y género.
En términos generales, ambos grupos describen la desigualdad entre hombres y mujeres en cuanto a los roles en el hogar. Las dos agrupaciones identifican a la madre como la persona que asume la carga de limpieza del hogar, el cuido de los hijos, etc., situación a la que no escapan algunas de las adolescentes participantes de este proyecto, como señalaremos más adelante.

Existe, además, el reclamo de otros espacios, sobre todo para aquellas niñas y adolescentes que practican algún deporte. En el caso de las niñas de Liga Fem: la cancha para nosotras de Sepro Joven, es necesario resaltar que lo vivido ellas en dicho proyecto ha abonado, visiblemente, a sus propios procesos de empoderamiento. Estas niñas logran identificar (y expresar abiertamente) los prejuicios que enfrentan las mujeres, cuando se trata del fútbol u otros deportes. Sin embargo, y aún más gratificante, es ver como establecen reglas, comparten el liderazgo y se posicionan de forma crítica ante los problemas comunitarios identificados.

Por otro lado, encontramos, en el caso de las adolescentes, no solo el reclamo al poco o nulo espacio que tienen las mujeres para practicar al fútbol, sino que también deben enfrentar el acoso por parte de sus pares, como sucede a una adolescente, quien comparte su experiencia en el colegio, donde, en repetidas ocasiones, le han cuestionado su 
identidad sexual, porque le gusta practicar este deporte.

No obstante, estas manifestaciones sexistas pasan igual inversamente; tal es el caso de uno de los adolescentes, quien nos comenta que, por tocar la lira en la banda del colegio, es molestado por algunos de sus pares, al ser considerada un instrumento para "chicas". Estos ejemplos expuestos por las personas participantes reflejan la discriminación que viven por el sexismo enraizado en nuestras casas, centros educativos y comunidades. Tal sexismo lo entendemos como "el conjunto de actitudes y creencias que convierten al sexo o género de las personas en el elemento determinante para atribuirles o dejar de reconocerles valor, capacidades o méritos particulares" (Obando, 2011: p. 229).

Otras formas más sutiles de violencia estructural que viven las adolescentes fueron identificadas por las facilitadoras, no solo en términos de las narrativas de los participantes, sino en algunas situaciones que se presentaron a lo largo del proceso. Un ejemplo de ello fue la justificación de las ausencias a las sesiones, las cuales, en el caso de las adolescentes, ocurrían mayormente, en términos de sus "responsabilidades domésticas", ellas tenían que quedarse en casa cuidando a sus hermanos menores o encargándose de las labores del hogar, mientras la mamá salía a trabajar o a hacer mandados. Contrariamente, los hombres, sus ausencias se justificaban en razón de otras actividades, como el fútbol, la banda del colegio o por situaciones económicas ("mi mamá no tenía dinero para el pasaje del bus").

La carga doméstica que se traslada a niñas y adolescentes interfiere en el pleno desarrollo de estas, no solo al limitar su participación en programas de educación no formal como este proyecto, sino, incluso, afectando su proceso formativo, ya que, con mayor frecuencia, que tienen que asumir estas responsabilidades faltan a clases, o carecen de tiempo y energía para estudiar, hacer sus tareas, etc. De esta forma, el derecho a la educación ${ }^{8}$ se ve vulnerado y tiene un impacto negativo en el desarrollo integral de las mujeres.

Aunado a lo dicho en párrafos anteriores, se evidencia cómo las personas participantes, si bien reconocen formas de agresión contra las mujeres, como la violencia sexual o el acoso callejero, también, en sus interpretaciones y reflexiones, se encuentran patrones

8 La UNESCO señala que "la educación capacita a las mujeres para vencer la discriminación. Las niñas y las jóvenes que han recibido educación conocen mejor sus derechos, y tienen mayor confianza y libertad para tomar decisiones que afectan a su vida, mejorar la salud y las posibilidades de supervivencia tanto propias como de sus hijos, y acrecentar sus perspectivas de trabajo. Lograr que las niñas sigan en la escuela es uno de los medios más eficaces de evitar el matrimonio y la maternidad precoces de las menores. La educación es también un factor fundamental para acelerar la transición demográfica a tasas de natalidad y mortalidad más bajas" (pág. 3)
192 Proyecto Construcción de Zonas de Paz: análisis de la percepción de la niñez y la adolescencia sobre la violencia y la paz en la comunidad de Guararí López-Céspedes/ Guzmán-Sierra 
y discursos aprendidos de tinte misógino y patriarcalista, que justifican esa violencia. Por ejemplo, algunas de las jóvenes consideran que la forma en que las chicas visten "provoca" que los hombres les digan cosas en la calle.

Entre los participantes masculinos, hubo quienes señalaron que los celos de los hombres, tanto como el consumo de alcohol, son causas de abusos físicos y psicológicos, incluso resaltando casos en los que ellos dejan a las mujeres encerradas en la casa $y$, si salen, las agreden físicamente.

Las múltiples formas de violencia que han experimentado las personas participantes se potencian como consecuencia del hacinamiento en el que viven, las estructuras de las casas y la cercanía, como nos señala una de las implicadas; permiten que se escuchen gritos, malos tratos, abuso físico, etc., que recibe su vecina por parte de su pareja?

El espacio se convierte en contenedor y naturalizador de la violencia, las personas la viven de forma directa o indirecta, sin que se tenga claridad de cómo

9 En relación con los asentamientos humanos y el alberge, IASC señala: "La ocupación excesiva, la falta de intimidad o cerrojos, una iluminación insuficiente, una mala distribución de los artículos no alimentarios y la construcción de albergues en lugares poco seguros incrementan el riesgo de violencia doméstica, agresión sexual, acoso, explotación sexual y otras formas de violencia de género, de las que son víctimas especialmente las mujeres y las niñas" (IASC, 2015, p. iii). reaccionar a esta. Se establece el silencio como elemento normalizador $y$, a su vez, controlador de las acciones. Ante los gritos de maltrato de una vecina, por los abusos de su esposo, aparece el silencio, esta manera establecida de "responder" a la violencia que se evidencia en otras expresiones, por ejemplo, cuando hay balaceras o robos. De acuerdo con el Fondo de Población de las $\mathrm{Na}$ ciones Unidas (UNFPA) ${ }^{10}$, en las áreas urbanas, las mujeres pueden correr un riesgo mayor de violencia basado en el género, frente a la menor probabilidad de que intervengan los vecinos.

La violencia contra las mujeres no es ajena a las vivencias de las personas participantes, quienes, en sus narraciones, las ubican desde los espacios comunitarios hasta los más íntimos y familiares. No pareciera ser casualidad que, en la cartografía simbólica realizada por las personas involucradas, mientras que las niñas hablaban de sus casas como lugares de paz, contrariamente, los adolescentes identificaron solo sus habitaciones como e ambiente pacífico libre de violencia. Otro elemento resaltado, principalmente por las adolescentes, fue el de la hipersexualización femenina y los estereotipos estéticos sobre los cuerpos de las mujeres.

10 Ver ONU-HÁBITAT, Igualdad de Género para Ciudades más Inteligentes. Desafíos y Avances, p. 12. 
El bombardeo mediático constante que nos vende la idea de corporeidad femenina con características que podríamos incluso señalar como lejanas a las formas en que esta se constituye en nuestra sociedad latinoamericana; desde el color de la piel, el cabello o los ojos, hasta el tamaño las caderas o la estatura contribuye a la continua laceración con que las mujeres dialogan con sus cuerpos.

El manejo obsesivo del cuerpo, en términos de ese canon de belleza, genera entre estas jóvenes luchas internas, cuando dicho canon no las representa o deben soportar las burlas que reciben de sus pares por su apariencia física. Una de las jóvenes, en su reflexión sobre las diversas formas de violencia, señaló que existe una "discriminación por obesidad”. Al ser consultada sobre ello, narró las maneras en que las mujeres "obesas" reciben mofas constantes y cómo esto puede llevarlas a la bulimia o la anorexia.

Se identifica también un discurso de las participantes adolescentes, que evidencia la construcción de patrones de dependencia afectiva con la pareja. Este sentimiento se alimenta de las canciones que cantan y bailan; los personajes femeninos de las novelas mexicanas y colombianas que tanto les gusta ver a algunas de ellas; las experiencias de vida de sus madres, hermanas, tías, amigas, las cuales se ven reflejadas en las actividades desarrolladas en las sesiones. Co- mentarios como "se siente sola porque la dejó el novio", de una adolescente de 14 años, o "mi papá dejó a mi mamá", "la abandonó", han sido recurrentes durante el proceso. En este sentido, se evidencia cómo la construcción de lazos afectivos con posibles parejas, amistades y pares se da desde la carencia y el miedo a ser abandonadas, situación que puede empujar a las mujeres a asumir roles sumisos y de subordinación en sus relaciones.

En resumen, la violencia contra las mujeres en sus múltiples expresiones (física, sexual, psicológica, patrimonial, estructural, etc.) estuvo presente en todas las cartografías simbólicas realizadas por las personas participantes, a diferencia de otras formas de agresión, como la ejercida contra el ambiente. Sus relatos ilustran las maneras más crueles en que estos actos violetos atraviesan los cuerpos de las mujeres y, en sentido más amplio, los de todos los involucrados en el proyecto, hombres y mujeres, quienes conviven en estos espacios y se alimentan de ellas.

Pensar la paz desde la abstracción puede llevarnos a idealizaciones, estados de pureza, imposibles de alcanzar para los seres humanos o a invisibilizar y desvalorizar aquellas acciones que nos permiten construir, desde nuestras subjetividades, formas de convivencia pacífica. La paz o "las paces" se construyen a través de procesos tanto personales 
como colectivos, desde los espacios más íntimos y minúsculos hasta aquellos que se extienden a lo público, local, comunitario. La construcción de la paz es, en todo caso, un ejercicio en el cual participan todas las personas de la comunidad, entendida esta no como el barrio, sino como colectivos de seres humanos que conviven en ambientes diversos, donde se construye una identidad común asociada a valores comunes.

En ese sentido, uno de los objetivos de este proyecto está dirigido a que las personas participantes no solamente identifiquen formas de violencia, sino que también reconozcan mecanismos de paz presentes en sus comunidades, partiendo de la premisa de que no todo es completamente negativo o violento en determinados espacios, a pesar de los estereotipos y estigmas asociados a las personas de Guararí y el reforzamiento de estos, por parte de los medios de comunicación y otras instituciones. Dichos estereotipos son, ampliamente, reconocidos por los participantes, incluso, para uno de los adolescentes, participar en el Proyecto Construcción de Zonas de Paz es una manera de dar a conocer que en Guararí hay gente educada, trabajadora, "gente buena" (adolescente, 13 años).

Del proceso vivido, sobresalen 2 elementos de análisis importantes para nuestra reflexión, sobre las percepciones de las personas participantes. Primero, efectivamente, como se señala en el párrafo anterior, tanto las niñas como los adolescentes, reconocen los espacios como lugares donde, si bien es cierto existen muchas expresiones de violencia, se identifican formas de vivir la paz. Esta vivencia se identifica desde lo cotidiano y rompe con ideas erróneas de que la paz es la ausencia de violencia o como idealización abstracta. De tal modo, las personas participantes expresan sensaciones de paz, tanto de una planta de guineo sembrada en una acera, frente a la casa de unas de las participantes, como de espacios más estructurados como la escuela de la comunidad.

Un segundo elemento por subrayar sería la conceptualización de la paz, hecha por las niñas y los adolescentes. De esta forma, en la tarea de construir nociones pacíficas, de paz, las personas participantes reconocen espacios donde vivirlas, aquellos entornos que les protegen y garantizan experimentar plenamente sus derechos humanos, así la escuela o el centro de salud representan, respectivamente, un sitio para aprender (derecho a la educación) y otro que les cuida cuando están enfermas (derecho a la salud). Esto se puede evidenciar en más ambientes identificados por los involucrados en el estudio: la verdulería, que les brinda verduras y frutas (derecho a una alimentación sana) o el parque infantil, asociado a su derecho a la recreación. 
Aunado a lo anterior, las personas participantes reconocen los espacios como garantes de derechos, no solo en el plano personal, sino a partir de las necesidades de otros integrantes de la comunidad. Un ejemplo de esto es que identifican el servicio de buses públicos como lugar de paz, no solamente porque en ellos pueden desplazarse a diversas localidades, también con especial relevancia, señalan que les generan paz los buses que tienen rampas para personas usuarias de silla de ruedas. Lo mismo sucede con la Casa de la Mujer, identificada como un sitio que ayuda a la población femenina de la comunidad.

Tanto las niñas como los adolescentes participantes consideran en el Centro Cívico por la Paz (CCP) un espacio donde hallan formas pacíficas de convivencia. Se evidencia un ejercicio de apropiación de los entornos ofrecidos por el CCP, como las áreas para practicar deportes como el fútbol o espacios para leer como la biblioteca. Una de las participantes nos dice: "a mí la biblioteca me gusta porque aprendo, y puedo tener paz leyendo" (niña, 7 años).

Finalmente, el CCP se constituye en un espacio donde las personas menores de edad pueden vincularse con sus pares, construir relaciones afectivas desde el respeto, la solidaridad y la igualdad. Es un lugar que les permite abstraerse de la violencia normalizada que ven día tras día, ya sea en la pulpería o en la esquina, donde asaltan con armas de fuego, en el sitio donde venden drogas o en la casa donde gritan y golpean. Una de las niñas refiere al CCP de la siguiente forma: "Yo acá tengo las posibilidades de compartir con las personas, cuando estoy triste y cuando estoy feliz" (niña, 9 años)

Las repercusiones de la violencia en las vidas de niñez y adolescencia de Guararí tienen un alto coste personal, familiar y social, en términos de afectaciones psicosociales tales como depresión, ansiedad, hiperactividad, incremento en conductas agresivas, bajo rendimiento escolar, consumo de alcohol u otras drogas, vinculación con pandillas, explotación sexual comercial, trata de personas, entre otros, que mantienen los ciclos de violencia, perpetuando la pobreza y exclusión social.

A partir de la narración de sus experiencias y percepciones en torno a la violencia, podemos discurrir el estado de vulnerabilidad en que se encuentran muchas de estas personas, principalmente, si consideramos que bastantes viven formas de violencia en espacios que tendrían que ser seguros, como lo es su propio hogar. Al ser este último determinante en el desarrollo de dichas conductas, familias que enfrentan los conflictos con golpes o gritos, que carecen de herramientas para el diálogo, 
o construyen vínculos deficientes, poco afectivos, incrementan las posibilidades de que la juventud quede atrapada en estos ciclos violentos.

Es importante insistir, como se adelantó, en que la construcción de la personalidad y la identidad de estas personas está marcada por la desigualdad, la discriminación y la estigmatización, por tanto la tendencia a conductas violentas pareciera ser una forma de caparazón ante las agresiones que se les imponen. La violencia de un medio ambiente y de un espacio urbano tanto contaminado como desordenado se impone y suma a la escasez económica, la carencia de recursos, los pocos estímulos y la falta de atención institucional. Esto decanta en un creciente descontento y una desilusión de las posibilidades comunitarias de gestión y así como de las buenas intenciones de los servicios estatales; tales emociones se hacen más palpables a mayor edad de las personas participantes. Esta situación preocupa aún más, cuando pareciera inhibir las probabilidades creativas e imaginativas para edificar comunidades menos violentas.

En este sentido, el CCP logra su objetivo de convertirse en un centro de paz y generación de alternativas para las personas menores de edad. La construcción de una ciudadanía activa y positiva es un reto fundamental de dicho centro en Guararí, el cual, de alguna manera, abarcaría los vacíos de la institucionali- dad. Los participantes del proyecto han encontrado en el CCP un espacio donde se construyen como sujetos de derechos, críticos y reflexivos de su realidad, la de sus familias, la de la comunidad y la del país. Así mismo, aprenden a convivir en un ambiente horizontal y respetuoso e interdependiente, abonando al tejido social.

Finalmente, los participantes son conscientes de que la paz no es un absoluto y lo demuestran en las capturas de sus fotografías, este valor se entrelaza y convive con la violencia; sin embargo, ellos entienden esta complejidad desde lo cotidiano, encontrando formas pacíficas de convivencia en los más pequeños elementos. En ese sentido, tanto las facilitadoras como los estudiantes de la Maestría de Derechos Humanos y Educación para la Paz del IDELA caminamos con las niñas del programa Sepro Joven y con los adolescentes del Proyecto Construyendo Zonas de Paz, por su comunidad, y, en ese caminar, encontramos, en esta población creativa e imaginativa, un ejercicio de profunda reflexión sobre este espacio, Guararí, desde principios afectivos, solidarios y colaborativos, el diálogo, el juego, la risa, el abrazo. 


\section{Referencias bibliográficas}

Bauman, Z. (2001). La globalización, consecuencias humanas. México: FCE.

Chaves, L. (2014). Diagnóstico 2014. Heredia: Centro Cívico por la $\mathrm{Paz}$ Guararí. (Documento interno de trabajo).

De Sousa Santos, B. (2006). Conocer desde el Sur. Para una cultura política emancipadora. Perú: UNMSM.

Galtung, J. (1998). Tras la violencias, 3R: reconstrucción, reconciliación, resolución. Afrontando los efectos visibles e invisibles de la violencia. España: Gernika Gogoratuz.

Grillo, M. (2014). Modelo base de atención Centros Cívicos por la Paz (CCP). San José: Ministerio de Justicia y Paz.

Herrera Flores, J. (2008). La reinvención de los derechos humanos. Andalucía: Editorial Atrapasueños.

IASC. (2015). Guía temática. Directrices para la integración de las intervenciones contra la violencia de género en la acción humanitaria: Reducir el riesgo, promover la resiliencia e impulsar la recuperación. Recuperado: https://gbvguidelines.org/wp/ wp-content/uploads/2016/03/ GBV_TAG_SPANISH_SHELTER-021916.pdf
Lederach, J. P. (2007). La imaginación moral. El arte y el alma de la construcción de la paz. Bilbao: Gernika Gogoratuz.

Lopez, S. (2011). Prácticas de justicia restaurativa para una cultura de paz. En UPAZ (Ed.) Desarrollo Profesional para Construir una Cultura de Paz (pp. 309-406). San José: UPAZ .

Obando, A. (2011). Educación género sensitiva: Paradigma de una cultura de paz. En UPAZ (Ed.) Desarrollo profesional para construir una cultura de paz. San José: UPAZ.

ONU-HÁBITAT. (2010). Igualdad de Género para Ciudades más Inteligentes. Desafíos y Avances. Nairobi.

UNESCO. (2013). Informe de Seguimiento de la Educacion para Todos en el Mundo. Ficha Descriptiva. Recuperado de: https:// es.unesco.org/gem-report/ sites/gem-report/files/girls-factsheet-sp2.pdf. 